\title{
Prevalence of human papillomavirus infection in Argentinean women attending two different hospitals prior to the implementation of the National vaccination program
}

\author{
Diego Chouhy ${ }^{1,2}$, Rubén Mamprín D’Andrea ${ }^{3}$, Mercedes Iglesias ${ }^{4}$, Analía Messina ${ }^{5}$, Juan J. \\ Ivancovich $^{6}$, Belen Cerda ${ }^{1}$, Diana Galimberti ${ }^{5}$, Hebe Bottai ${ }^{6}$, and Adriana A. Giri ${ }^{1,2, \#}$ \\ ${ }^{1}$ Area Virología, Facultad de Ciencias Bioquímicas y Farmacéuticas, Universidad Nacional de \\ Rosario. Rosario, Argentina \\ 2Instituto de Biología Molecular y Celular de Rosario. Rosario, Argentina \\ ${ }^{3}$ División de Ginecología, Hospital-Escuela "Eva Perón”. Granadero Baigorria, Argentina \\ ${ }^{4}$ Laboratorio Central, Hospital General de Agudos "Dr. Teodoro Alvarez". Buenos Aires, \\ Argentina \\ ${ }^{5}$ Departamento de Obstetricia y Pediatría, Hospital General de Agudos "Dr. Teodoro Alvarez". \\ Buenos Aires, Argentina \\ ${ }^{6}$ Área Estadística y Procesamiento de Datos, Facultad de Ciencias Bioquímicas y Farmacéuticas, \\ Universidad Nacional de Rosario. Rosario, Argentina
}

\section{Abstract}

Cervarix vaccine was included in the National Immunization Program of Argentina in 2011 but data about the local distribution of human papillomavirus (HPV) infection in women exposed to the virus are scarce. This cross-sectional study determined the prevalence and type distribution of HPV infection in unvaccinated women attending routine gynaecological screening in two public hospitals located in Buenos Aires and Santa Fe, Argentina. Socio-demographic, sexual behaviour and co-factors information was obtained from all participants (Buenos Aires, $n=429$; Santa Fe, $n=433$ ). Cervicovaginal swabs were tested with an MY11/09 primer-based assay and with the CUT primer system targeting mucosal/cutaneous HPVs.

Participants from Buenos Aires showed significantly higher rates of HPV infection (52.4\% vs. $40.6 \%$ ), of multiple infections (24.2\% vs. $16.4 \%)$, and of low-risk (20.3\% vs. $13.9 \%)$ and highrisk types (44.1\% vs. 33.3\%) than those from Santa Fe. HPV-66 (Buenos Aires: 17\%) and HPV-16 (Santa Fe: 8.5\%) were the most prevalent types. Novel HPV-66 putative subtype and variants were identified. Vaccine types 16 and 18 were frequent (Buenos Aires: 13.5\%; Santa Fe F: $10.2 \%$ ) but few participants had co-infections with both (Buenos Aires: 1.4\%; Santa Fe: $0.2 \%$ ). A common risk factor for HPV infection was having a new sexual partner in the last year (Buenos Aires: OR 2.53, $p<0.001$; Santa Fe: OR 1.85, $p=0.04)$. This study provides valuable baseline data for future assessment of the impact of massive vaccination in Argentina and it underlines the use of additional HPV testing strategies, such as the CUT system, for surveillance and vaccinology.

\footnotetext{
\#Correspondence to: Adriana A. Giri, Professor. Área Virología, Instituto de Biología Molecular y Celular de Rosario (CONICET) Facultad de Ciencias Bioquímicas y Farmacéuticas, Universidad Nacional de Rosario. Address: Suipacha 531, (S2002) Rosario, Argentina.giri@ibr.gov.ar.

The authors declare that they have no competing interests.
} 


\section{Keywords}

cervical HPV infection; prevaccination; Argentina; MY11/09 and CUT primers; HPV-66 diversity

\section{INTRODUCTION}

Human papillomavirus (HPV) infection is a well-established cause of cervical cancer and there is growing evidence that HPV is a relevant factor in other anogenital and head and neck cancers [zur Hausen, 2002]. HPVs are DNA viruses perfectly adapted to the differentiating epithelial cells of skin or mucosa, which exploit the cellular machinery for their own purposes [Longworth and Laimins, 2004].

HPVs have been divided into 5 genera (Alpha, Beta, Gamma, $\mathrm{Mu}$, and $\mathrm{Nu}$ ) according to the phylogenetic relationships of their complete L1 gene sequences [de Villiers et al., 2004]. More than $40 \mathrm{HPV}$ types have been identified infecting the genital tract and they have been grouped into the Alpha genus [de Villiers et al., 2004]. In addition, genital HPVs have been divided into low-risk (LR) and high-risk (HR) HPV types depending upon their association with the development of benign (condylomas) and malignant anogenital lesions, respectively [zur Hausen, 2002; Munoz et al., 2003].

Cervical cancer is the second most common cancer among women worldwide, with an estimated 529,000 new cases and 275,000 deaths in 2008 [WHO/ICO, 2010]. About $86 \%$ of the cases occur in developing countries, accounting for $13 \%$ of all cancers affecting women. South America has some of the highest cervical cancer incidence and mortality rates in the world, surpassed only by most regions of Africa and South-Central Asia [Ferlay et al., 2010]. An estimation for 2008 showed Argentina as one of the countries with the lowest incidence $(17.5 / 100,000)$ and mortality $(7.4 / 100,000)$ rates in South America $(24.1 / 100,000$ and 10.8/100,000, respectively) [WHO/ICO, 2010]. Cervical cancer ranks as the third most frequent cancer among Argentinean women and the second among women between 15 and 44 years old [WHO/ICO, 2010].

Active recruitment for cervical scrrening is recommended in the Argentinean National guidelines. However, the lack of population registries and postal systems deficiencies constitute an obstacle to increasing screening coverage through invitation letters, as implemented in several developed countries [Alliance for Cervical Cancer Prevention, 2004]. As a result, cervical screening in Argentina is opportunistic, performed mainly in urban areas and usually through family planning and reproductive health care facilities [Arrossi et al., 2010].

Two prophylactic HPV vaccines are currently being introduced in various countries: Gardasil (Merck, Whitehouse Station, NJ), and Cervarix (GlaxoSmithKline Biologicals, Rixensart, Belgium). Both vaccines offer protection against HR HPV types 16 and 18, which are estimated to account for $71 \%$ of invasive cervical cancers globally [de Sanjose et al., 2010]. Gardasil also offers protection for LR HPV types 6 and 11, which are responsible for $90 \%$ of all genital warts [Villa, 2007]. Both vaccines have been approved for use in Argentina, and Cervarix was included in the National Immunization Program in 2011.

About $20.1 \%$ of Argentinean women are estimated to harbour cervical HPV infection at any given time [WHO/ICO, 2010]. These estimates came from women with normal cytology residing in the cities of Concordia [Matos et al., 2003] and La Plata [Abba et al., 2003], with HPV prevalence values ranging from $16 \%$ to $46 \%$, respectively. These data indicate the heterogeneous character of HPV prevalence throughout the country and highlight the need for further studies about exposed women living in other areas of Argentina. 
HPV infection with genital types is a frequent sexually transmitted infection. In the vast majority of cases, these infections are usually transient and do not necessarily lead to clinically significant lesions of the cervical mucosa [Schiffman, 1994]. Therefore, understanding the local distribution of type-specific HPV infection in women exposed to the virus before the implementation of the vaccine provides valuable baseline data for future assessment of the impact of massive vaccination in Argentina.

This work reports the first pre-vaccine type-specific prevalence of HPV infection in sexually active women recruited during a routine gynaecological visit to two public hospitals located in Central Argentina. This analysis was performed using two HPV detection and typing methods: an MY11/09 primer-based assay [Chouhy et al., 2006], and the CUT primer system previously applied for the identification of a wide range of skin-associated HPVs from different genera [Chouhy et al., 2010]. The association between HPV infection and the socio-demographic characteristics, sexual behaviour and co-factors of the participants was explored.

\section{MATERIALS AND METHODS}

\section{Subject recruitment and data collection}

Between 2008 and 2010, sexually active women undergoing a routine gynaecological visit were recruited to participate in this cross-sectional study in two public hospitals located in Central Argentina: the Hospital-Escuela "Eva Perón", a provincial general hospital located in Granadero Baigorria city, province of Santa Fe, and the Hospital General de Agudos "Dr. Teodoro Alvarez", a municipal health centre located in Buenos Aires city. Both hospitals participate in the National Family Planning and Reproductive Health Program, receiving patients of median and low-income resources, with and without health insurance. The study was approved by the bioethics committees of each hospital, and written informed consents were obtained from all study participants. All procedures were in accordance with the Helsinki Declaration.

Women attending a routine gynaecological visit, who were non-postpartum for the past 6 weeks, were not hysterectomized, had no treatment for intraepithelial cervical disease or abnormal cytology within the past 18 months, and had not previously been vaccinated against HPV infection, were approached to participate in the study. After informed consent was obtained, a study questionnaire covering socio-demographics (age, country of birth, current residence, working status, education), sexual behaviour (age at first intercourse, lifetime number of sex partners, new sexual partner in the last year, contraceptive use and type), and co-factors related to the development of cervical cancer (history of sexually transmitted infections and tobacco use) was given by the interviewer [Almonte et al., 2008].

\section{Specimen collection and processing}

All women underwent a complete gynaecologic examination that included cervical cytology (Pap smear) and HPV DNA testing. For cervical cytology, an Ayres spatula was used to collect exocervical cells, and a conical cytobrush (Cervicep, Buenos Aires, Argentina) was used to collect endocervical cells. Once Pap smear was performed, the cytobrush was kept in $0.8 \mathrm{ml}$ PBS solution for HPV DNA testing and processed as has been previously described [Chouhy et al., 2006].

Cytological results were classified according to the Bethesda system 2001 [Solomon et al., 2002]. The following classes were distinguished: negative for intraepithelial lesions and malignancies (normal), atypical squamous cells of undetermined significance (ASC-US), low-grade squamous intraepithelial lesions (L-SIL), and high-grade squamous intraepithelial lesions (H-SIL). 


\section{HPV DNA testing}

HPV DNA detection and typing was done by two HPV DNA testing methods: the L1HPVPCR 16.4 assay, a modified version of a previously reported assay [Chouhy et al., 2006], and the CUT primer system [Chouhy et al., 2010]. A thermal cycler (Mastercycler Personal Eppendorf, Hamburg, Germany) was used in all the experiments involving PCR.

L1HPVPCR 16.4 assay-This MY11/MY09 primers-based test [Manos et al., 1989] was optimized for the simultaneous detection of HPV DNA and $\beta$-globin sequences in order to determine HPV status and sample integrity in a single reaction. MY11 and MY09 primers corresponded to nt 6,582-6,601 and 7,014-7,033, respectively, of the HPV-16 genome (AF125673). Briefly, $45 \mu \mathrm{l}$ of a PCR mixture containing $2.5 \mathrm{U}$ of Taq DNA polymerase (Fermentas, St. Leon-Rot, Germany), 1X PCR buffer, $2.75 \mathrm{mM} \mathrm{MgCl}, 0.3 \mathrm{mM}$ deoxynucleoside triphosphates, HPV amplification primers [0.8 $\mu \mathrm{M}$ of MY11, $0.4 \mu \mathrm{M}$ of each anti-sense MY09 and MYGC09 ( $5^{\prime}$-cgacccarwggaaaytgatc- $\left.3^{\prime}\right)$, both biotinylated at the $5^{\prime}$ ends] and $\beta$-globin amplification primers $(0.12 \mu \mathrm{M}$ of each PC04 and GH20) were prepared [Saiki et al., 1986]. A $5 \mu$ l aliquot of sample lysate was added to the PCR mixture and amplified using the following thermal profile: $2 \mathrm{~min}$ of initial denaturation at $95^{\circ} \mathrm{C}$ followed by 40 cycles of $40 \mathrm{~s}$ at $94^{\circ} \mathrm{C}, 40 \mathrm{~s}$ at $52^{\circ} \mathrm{C}, 40 \mathrm{~s}$ at $72^{\circ} \mathrm{C}$, and a final extension of 5 min at $72^{\circ} \mathrm{C}$. Each PCR run included the following controls: a positive control (a pool of 1,000-10,000 copies/reaction of plasmid controls for each HPV type analyzed in the assay), a negative control [5 ng of human placental DNA (Sigma, Buenos Aires, Argentina)] and a reagent control $\left(\mathrm{H}_{2} \mathrm{O}\right.$ instead of sample).

Amplicons $(10 \mu \mathrm{l})$ were first evaluated for $\beta$-globin and HPV bands with $2 \%$ agarose gel electrophoresis and ethidium bromide staining. Valid samples (positive for the $\beta$-globin gene) were hybridized with a generic probes cocktail, and detected colorimetrically to identify HPV infection with the method described earlier [Chouhy et al., 2006]. In this case, $2.5 \mu \mathrm{l}$ of each PCR product were added to $60 \mu \mathrm{l}$ of hybridization buffer containing $6 \mathrm{pmol}$ of each probe. The generic probes cocktail was a mixture of fluoresceinated GPX [Manos et al., 1989] and CHG (5' -ctgtwgtkgatacyacycgcagtac- $\left.3^{\prime}\right)$ probes. A sample was considered HPV-positive when its OD value after colorimetric detection was greater than the cut-off value determined as 2.5 times the OD value of the HPV-negative control of that experiment, as has been previously defined [Chouhy et al., 2006].

HPV-positive samples were typed for 14 HR HPV types (HPV types 16, 18, 31, 33, 35, 39, 45, 51, 52, 56, 58, 66, 68 and 73) and 2 LR HPV types (HPV types 6 and 11) using the same protocol described earlier [Chouhy et al., 2006]. Probes used for typing were the following: MYB-12 (HPV-6), MYB-13 (HPV-11), MYB95/MYB133 (HPV-16), WDB74/MYB130 (HPV-18), MYB128/AG111 (HPV-31), MYB16/MYB64 (HPV-33), MYB115/MY117 (HPV-35), MYB89/MYB90 (HPV-39), MYB69/MYB129_RC (HPV-45), BC51 (HPV-51), MYB81 (HPV-52), CHG56 (HPV-56), CHG58/MYB179 (HPV-58), MYB83 (HPV-66), MYB194/MYB191 (HPV-68) and CHG73 (HPV-73). BC51 (5'-cactgccactgctgcggtttc-3' ), C H G 56 ( $5^{\prime}$-gctaacctactggaggactgg-3' $)$, C H G 58 ( 5 '-gcactgaagtaactaaggaaggta- $\left.3^{\prime}\right)$ and CHG73 ( $5^{\prime}$-acaacgtatgccaactctaa- $\left.3^{\prime}\right)$ probes were designed for this work. AG111 was previously reported [Chouhy et al., 2006] and the rest were previously described [Manos et al., 1989]. One or two oligonucleotide probes per type were used (4 pmol of each typespecific probe). Hybridization temperatures in the liquid hybridization step were $55^{\circ} \mathrm{C}$ for HPV-31, HPV-33 and HPV-35, and $40^{\circ} \mathrm{C}$ for the rest. A sample was considered positive for a specific HPV type when its OD value after colorimetric detection was greater than the cutoff value determined as 4 times the OD value of the HPV-negative control, as previously defined [Chouhy et al., 2006]. 
CUT primer system-Suitable specimens were tested with CUT primers [forward and reverse CUT primers corresponded to nt 5,868-5,888 and 6,225-6,243, respectively, of the HPV-16 genome (AF125673)], with a "hanging droplet PCR" amplification strategy, and with HPV amplicon identification by electrophoresis in $2 \%$ agarose gel. Type determination was done by direct sequencing as previously described [Chouhy et al., 2010]. DNA sequencing was performed using sequencing facilities (Macrogen, Maryland Rockville, US) with reverse primer CUT1BRv. Electropherograms with multiple overlapping sequences were flagged as possible mixed infections. PCR products from such samples were cloned into pGEM-T-Easy vector (Promega, Buenos Aires, Argentina) and identified by PCR using M13 Fw/Rv primers. At least three recombinant clones were sequenced from each sample.

\section{Sequence analysis and accession numbers}

Sequences derived from the CUT amplification system were compared to available HPVsequences in the GenBank database using the BLAST server. The oncogenic risk associated to each HPV type was defined according to the current taxonomic classification [de Villiers et al., 2004; Schiffman et al., 2009]. Hence, types 16, 18, 26, 30, 31, 33, 35, 39, 45, 51, 52, $53,56,58,59,66,68,69,70,73$ and 82 were considered HR HPVs, while types $6,11,32$, $40,42,43,44,54,61,62,71,74,81,83,84,86,87,89,91,114$, and CP6108 were considered LR HPVs.

A novel putative HPV-66 subtype and variants were defined according to the current taxonomic classification [de Villiers et al., 2004]. CUT-fragments sequences showing sequence identities ranging from $90 \%$ to $98 \%$ were considered subtypes, and variants when showing identities ranging from $98 \%$ to $99 \%$ with respect to any of the previously known HPV-66 strains. In this analysis, full-length L1 ORF sequences from HPV-66 variants available at the GenBank were used for comparison [GenBank accession numbers U31794.1 (prototype) and EF177182.1 to EF177191.1].

HPV subtype and variants candidate sequences identified in this study have been submitted to the GenBank with the following accession numbers: RA-66b (JN122286), RA-66.1 (JN122285), RA-66.2 (JN122287), RA-66.3 (JN122288), RA-66.4 (JN122289), RA-66.5 (JN122290), RA-66.6 (JN122291) and RA-66.7 (JN122292).

\section{Statistical analyses}

Concordance between the CUT system and L1HPVPCR 16.4 assay for the total tested population was determined by the Kappa Coefficient $(K)$.

A descriptive analysis was done of the HPV infection and independent variables. Tests of association between HPV infection and its co-factors, the socio-demographic and behavioural characteristics, were based on the Wald $\chi^{2}$ statistics. Two separate logistic regressions were used, one for each site.

Characteristics with a significance level of $P<0.25$ based on Wald $\chi 2$ statistics, were considered in a multivariate model. In order to assess the most appropriate categorization of continuous variables that turned out significant in the multivariate logistic regression analysis, they were plotted against the log odds of HPV. The selection of variables in the final model was done using a purposeful selection of covariates based on the algorithm proposed by Hosmer and Lemeshow [Hosmer and Lemeshow, 2000]. Potential confounding variables and interactions were studied. Significance was evaluated at 0.1 alpha level. Model fit was assessed using the Le Cessie-van Houwelingen normal test statistics for the unweighted sum of squared errors. 
Statistical analyses were performed using R Development Core Team (2008). $P$-Values less than 0.05 were regarded statistically significant.

\section{RESULTS}

In the Santa Fe hospital, 472 healthy women were enrolled from September 2008 to June 2009. From them, 433 (92\%) samples suitable for PCR analysis were obtained. All participants with suitable samples were born in Argentina, with a mean age of 31.6 years (age range: 14 to 77 years), $10.6 \%$ were residing in Rosario city and $89.4 \%$ were living in 26 small cities distributed in the Southern Santa Fe province. Forty-one percent were employed and $56.3 \%$ had reached high school (studied for more than 7 years). Thirty-three percent were habitual tobacco users, and $9.5 \%$ reported a history of sexually transmitted infections. Cytological results for this site were: 403 normal (93.1\%), 14 ASC-US (3.2\%), 14 L-SIL $(3.2 \%)$ and $2 \mathrm{H}-\mathrm{SIL}(0.5 \%)$.

In the Buenos Aires hospital, 456 healthy women were recruited from March 2009 to August 2010; 429 of them (94\%) had suitable samples for PCR analysis. Participants had a mean age of 31.2 years (age range: 15 to 69 years), $81 \%$ were born in Argentina and 19\% in other South American countries (10\% in Bolivia, 7\% in Paraguay, 1.5\% in Dominican Republic and $0.5 \%$ in Peru). Eighty percent were inhabitants of Buenos Aires City and 20\% resided in the surrounding conurbation in the province of Buenos Aires. Fifty-five percent were employed and $70 \%$ had reached high school. Twenty-two percent were habitual tobacco users, and $9.3 \%$ reported a history of sexually transmitted infections. Cytological results for this group of women were: 371 normal (86.5\%), 23 ASC-US (5.4\%), 32 L-SIL (7.4\%) and $3 \mathrm{H}-\mathrm{SIL}(0.7 \%)$.

Significant differences were found among women from each hospital regarding education ( $p$ $<0.001)$, working status $(p<0.001)$, age of first sexual intercourse $(p<0.001)$, number of lifetime sexual partners $(p=0.02)$, contraception method used $(p<0.001)$, tobacco use ( $p<$ $0.001)$ and cytological results $(p=0.01)$. Yet, women from both sites showed similarities for variables associated with an increased risk of HPV infection such as mean age $(p=0.51)$, new sexual partner in the last year $(p=0.23)$, and history of sexually transmitted infections $(p=0.94)$. Detailed data on the characteristics of women from each site is available from the authors.

\section{HPV DNA test results and comparison}

The frequency of HPV infection in the total tested population $(n=862)$ was determined through hybridization with generic HPV probes and colorimetric detection for MY-derived amplification (L1HPVPCR 16.4 assay), or by the presence of visible bands after electrophoresis in 2\% agarose gel (CUT system). HPV DNA was present in $46.5 \%$ of the analyzed women (401/862). HPV DNA was detected more often by CUT $(336 / 862)$ than by MY primers (245/862) and this difference was found statistically significant $(p<0.0001)$. In addition, 180 women were positive and 461 negative for HPV DNA by both tests, leading to a general concordance of $74.4 \%$ (641/862) ( $K=0.43$; 95\% CI, 0.37-0.49). HPV types were determined by hybridization to type-specific probes (L1HPVPCR 16.4 assay) or by direct sequencing (CUT system). At least one HPV type common to 123 women was found, leading to a type-specific concordance of $68.3 \%$ (123/180) between both tests.

The capacity of each HPV DNA testing strategy for detecting HPV types from different species within the Alpha genus is summarized in Table I. Overall, both assays detected 743 viruses corresponding to $40 \mathrm{HPV}$ types, $21 \mathrm{HR}$ (species 5, 6, 7, 9 and 11) and $19 \mathrm{LR}$ HPV types (species 1, 3, 8,10,13 and 14). Altogether, these results indicate that both assays used 
in combination comprehensively cover all mucosal HPV types involved in genital infections.

\section{Overall HPV DNA prevalence and factors associated with HPV infection}

HPV frequencies were explored using both HPV testing strategies in combination. Table II shows the socio-demographic and sexual behaviour information and related co-factors associated with HPV infection in this study or according to others [Almonte et al., 2008]. Participants from Buenos Aires showed a significantly higher rate of HPV infection with respect to those from Santa Fe $(52.4 \%$ Vs $40.6 \%, p<0.001)$. Regarding age groups, women aged 15-23 years showed the highest HPV prevalence in Buenos Aires $(64.2 \%, p=0.02)$. In contrast, women aged 31-47 years showed the lowest rates of HPV infection in Santa Fe $(31.3 \%, p=0.03)$, with an increasing trend in the lower and upper age intervals. Women who had more than 3 sexual partners in their lifetime (Buenos Aires, $p=0.07$; Santa Fe, $p=$ 0.001 ), and those who had a new sexual partner in the last year (Buenos Aires, $p<0.001$; Santa Fe, $p=0.001$ ) showed higher rates of HPV infection. On the other hand, HPV prevalence in both sites did not vary significantly with respect to other socio-demographics (education and working status), nor to age at first sexual intercourse, nor to the kind of contraceptive used or to co-factors related to the development of cervical cancer.

Those variables associated with HPV DNA detection at $p<0.25$ in the univariate analysis were examined with the multivariate logistic regression analysis (Table II). According to the multivariate analysis (Table III), only women aged below 24 years and those who had a new sexual partner in the last year showed a higher chance of HPV infection in Buenos Aires. For women from Santa Fe, only one variable was associated with HPV infection after multivariate analysis (Table III): women who had a new sexual partner in the last year had a higher chance of HPV infection than those that did not. The variable "number of lifetime sexual partners" remained in the final model because it was found to be slightly confounding.

\section{HPV type distribution according to cytology by site}

Table IV shows the overall distribution of HPV genotypes in each hospital according to cytology. In general, women from Buenos Aires showed higher frequencies and wider distribution of HPV genotypes than those from Santa Fe. HPV prevalence and HR HPV prevalence increased according to cytological diagnosis severity in both hospitals. HPV-66 was the most prevalent type overall (17\%) and also in most cytological diagnosis in Buenos Aires, whereas HPV-16 (8.5\%) was the most common type overall in Santa Fe.

With respect to LR HPV types, HPV-43 (3.7\%) was the most common type in Buenos Aires, while no particular type predominated in Santa Fe. With respect to the most common types detected in each site, HR HPV types 16, 31, 39, 52, 56, 58 and 66 were found in both sites but with different frequencies (Fig. 1). HPV-18 and HPV-68 were among the most common types in Buenos Aires (Fig. 1A) but they did not appear in Santa Fe (Fig. 1B). The same was true for HR HPV types 33 and 51 in Santa Fe (Fig. 1). There was a statistically significant difference in the frequency of HPV-66 between hospitals (Buenos Aires: 17\%; Santa Fe: $7.2 \% ; p=0.0001)$.

Frequencies of specific types and multiple infections in each hospital are shown in Fig. 2. Overall, women from Buenos Aires showed higher frequencies with respect to those from Santa Fe regarding HPV infection $(52.4 \%$ vs. $40.6 \% ; p<0.001)$, multiple infections $(24.2 \%$ vs. $16.4 \% ; p=0.005)$, HR HPV types $(44.1 \%$ vs. $33.3 \% ; p=0.001)$ and LR HPV types (20.3\% vs. $13.9 \% ; p=0.01)$. Participants from Buenos Aires also showed higher frequencies of the vaccinal types $6 / 11 / 16 / 18$ (15.4\% vs. 12.9\%) and of the 5 most 
carcinogenic types 16/18/31/33/45 (19.3\% vs. 17.6\%) with respect to those from Santa Fe, but these differences were not found to be statistically significant.

Most participants contained a single HPV type [Buenos Aires: 28.2\% (121/429); Santa Fe: $24.2 \%(105 / 433)]$ while most co-infections involved 2 HPV genotypes [Buenos Aires: $11.9 \%$ (51/429); Santa Fe: 8.3\% (36/433)] in both sites. However, multiple infections with 3 HPV types [Buenos Aires: 5.6\% (24/429); Santa Fe: 5.1\% (22/433)] and with 4 or more HPV genotypes [Buenos Aires: 6.7\% (29/429); Santa Fe: 3.0\% (13/433)] were also common in both hospitals.

Overall, vaccinal HPV types 16 and/or 18 were present as single infections and also in combination with other HPV types in women from both sites [Buenos Aires: 13.5\% (58/429); Santa Fe: 10.2\% (44/433)]. However, few participants at each hospital had HPV types 16/18 included in Cervarix [Buenos Aires: 1.4\% (6/429); Santa Fe: 0.2\% (1/433)] and none had all HPV types 6/11/16/18 included in Gardasil.

\section{HPV-66 Genomic Diversity}

In view of the high prevalence of HPV-66 found in this study, the genomic diversity of the CUT fragments identified in each site was explored. Ninety HPV-66 CUT fragments with clear cromatograms were selected from samples presenting single infections, or multiple infections that were subsequently cloned and sequenced.

Sequence comparisons of the HPV-66 CUT fragments revealed that 20/90 sequences (18 from Buenos Aires; 2 from Santa Fe) showed complete sequence identity to the HPV-66 prototype (U31794.1), while 54/90 sequences ( 32 from Buenos Aires; 22 from Santa Fe) had complete sequence identity to the EF177182.1 variant. In the remaining 16/90 sequences, the first putative subtype and 7 novel putative variants were identified (Fig. 3A).

The putative subtype (RA-66b) was detected in a cervical sample from a 26-year old Bolivian woman with normal cytology (patient BA035) recruited at the Buenos Aires hospital. RA-66b showed nucleotide identities ranging from $90.7 \%$ to $92.2 \%$ (Fig. 3B), resulting in 6 out of 89 (6.7\%) amino acid changes (data not shown). The novel HPV-66 putative variants (isolates RA-66.1 to RA-66.7) showed nucleotide identities ranging from $96.3 \%$ to $99.6 \%$. Of them, 2 putative variants were found in 3 women from Santa Fe (RA-66.1, RA-66.3), 3 in 4 women from Buenos Aires (RA-66.4, RA-66.5, RA-66.7), and 2 in 6 women from both sites (RA-66.2, RA-66.6). The isolate RA-66.6 was the most common HPV-66 putative variant. Finally, mixed infections in patients BA032 (RA-66.4, RA-66.5) and SF241 (RA-66.2, RA-66.6) were also detected.

\section{DISCUSSION}

This was the first large-scale study to estimate the pre-vaccine HPV type-specific prevalence in sexually active women attending a routine gynaecological visit in two hospitals located in the most populated regions of Argentina. Since recruitment for the study was hospital-based, the sample populations were not representative of the general population. However, given that Argentina is a federation in which each province autonomously establishes its own health policies such as funding and management of the health care services [Arrossi et al., 2010], these results depict routine clinical practice in public hospitals of Argentina.

This investigation was done using two HPV testing strategies in combination: an MY09/11based test [Chouhy et al., 2006] and the CUT system [Chouhy et al., 2010]. The capacity of each test for detecting HPV types was determined with respect to the total tested population. The L1HPVPCR 16.4 assay detected 472 viruses corresponding to the 16 genotypes 
included in the assay, while the CUT system identified 401 viruses corresponding to 36 genotypes (17 HR and 19 LR HPV types, Table I). In addition, only 130 viruses (17.5\%) were simultaneously detected by both tests, indicating that the assays used in this study complement each other and can be used in combination to explore the HPV types involved in cervix infections.

The behaviour of HPV in the cervix is strongly correlated with phylogenetic categories [Schiffman et al., 2009], and all HPV genotypes infecting the cervical mucosa are included in the Alpha genus [de Villiers et al., 2004]. HR HPV types have been grouped into species 5, 6, 7, 9 and 11 while LR HPV types are grouped into species 1, 3, 8, 10,13 and 14. The CUT system identified viruses from 10 out of the 11 species containing mucosal types (Table I). These results, together with the previous application of this system for identifying skin-associated HPVs from Alpha-, Beta-, and Gamma-PV genera [Chouhy et al., 2010], indicate that the CUT system is one of the up-to-date primers with the broadest capacity for the detection of HPVs. This characteristic makes the CUT system an ideal tool to investigate the epidemiology of HPV in different epithelia.

Several international studies have examined the prevalence of HPV in women. The diversity of population samples, HPV amplification strategies and typing methods make it difficult to identify studies that are exactly comparable to each other [Bosch et al., 2008]. In order to avoid these limitations, identical study designs, recruitment protocols and HPV DNA testing strategies were applied in both hospitals to investigate the geographical differences in HPV prevalence and type distribution. With this approach, a significantly higher rate of HPV infection was found in women from Buenos Aires (52.4\%) than in those from Santa Fe $(40.6 \%)$. The same trend was observed in the frequencies of multiple infections, HR and LR HPV types (Fig. 2). The high rates of HPV prevalence found in participants from both health centres could be explained by the application of two HPV testing strategies in combination. In particular, the CUT system was able to identify HPV types that are not usually targeted in the assays used in most epidemiological studies (Table I) [Munoz et al., 2003; Bruni et al., 2010; Eklund et al., 2010]. Considering that Buenos Aires and Santa Fe hospitals are relatively close to each other (approximately $300 \mathrm{~km}$ ), these results reflect the heterogeneous nature of HPV prevalence throughout the country and are consistent with inter-regional variations reported for other parts of the world [Bosch et al., 2008; Bruni et al., 2010]. On the other hand, differences in the proportion of the cytological categories among women attending to each hospital (Table IV) could partly explain the higher HPV prevalence found in Buenos Aires. In fact, participants from Buenos Aires had a significantly higher proportion of cervical abnormalities (ASC-US, L-SIL, H-SIL), mainly due to the higher number of L-SIL cases $(p=0.01)$, than those from Santa Fe. Still, HPV infection rates differed significantly by site in women with normal cytology $(p=0.0005)$, the most represented cytological category (Table IV). Under these considerations, the significant differences found in HPV prevalence between the two hospitals may be due to the different lifestyles of big cities (Buenos Aires) and small towns (Santa Fe), as described before (e.g. age at first sexual intercourse, number of lifetime sexual partners, etc).

Because HPV is a sexually transmitted agent necessary for carcinogenesis, sexual behaviour, in combination with certain co-factors, is associated with an increased risk of infection [Almonte et al., 2008]. In this report, the variables associated with HPV infection that were significant in the univariate analysis in both sites were age and having new sex partners in the last year (Table II). In the multivariate analysis, having a new sexual partner in the last year remained a common risk factor for HPV infection in both hospitals (Table III). In addition, women more than 24 years old showed a higher chance of HPV infection in Buenos Aires. Nevertheless, the variables influencing HPV prevalence that were identified 
in each site were consistent with the typical risk factors associated with the acquisition of HPV [Almonte et al., 2008].

Considering that most women in both sites had normal cytology (Buenos Aires: 86.5\%, Santa Fe: 93.1\%) and the high HR-HPV rates found in this cytological category (Table IV), HPV DNA testing may be considered for molecular surveillance in Argentina. In addition, important differences related to HPV genotype distribution and frequencies were found between both sites (Fig. 1). The fact that HPV-16 was the most frequent type found in Santa Fe was predictable since it is the most common type everywhere and it also is the most prevalent type in normal cytology [Bruni et al., 2010] as well as in cervical lesions of different grade, including cervical cancer [de Sanjose et al., 2010]. However, finding HPV-66 as the most frequent type in Buenos Aires was an unexpected result. Moreover, the HPV-66 frequencies that were found in this study were among the highest reported worldwide [Bruni et al., 2010; de Sanjose et al., 2010], and also among the recently published prevalence studies in which this type was identified [Castellsague et al., 2012; da Silva et al., 2012; Mazarico and Gonzalez-Bosquet, 2012]. These findings are due to the application of the CUT primers that appeared to have a differential specificity with respect to the MY11/09 primers (100/104 Vs 38/104, respectively) in detecting HPV-66 (Table I). In addition, a systematic mistyping between HPV-56 and HPV-66 has been reported for some commercial tests based on the MY11/09 primers and probes described by Manos and col. [Manos et al., 1989]; for example, the Linear Array assay (Roche Diagnostics, Basel, Switzerland) [Eklund et al., 2010]. This fact could affect the correct identification of the types that are important in HPV surveillance and vaccinology.

HPV-66 is an Alpha-PV from species 6 originally detected in a 38-year old patient biopsy with a stage I invasive squamous-cell carcinoma of the uterine cervix; it was classified as a member of an HPV group associated with genital neoplasia, together with HPV-56 [Tawheed et al., 1991]. However, HPV-66 was considered a "probable" high-risk type due to its low prevalence in cervical carcinomas [Munoz et al., 2003; Schiffman et al., 2009]. Based on the results obtained with the CUT system and the apparently lower efficiency of the MY11/09 primers-based assays in detecting this type (Table I), it can be speculated that HPV-66 frequency in epidemiological studies may be underestimated. On the other hand, HPV-66 is not included as a target virus in the commercially available Hybrid Capture II High-Risk HPV DNA Test (Qiagen, Hilden, Germany). These facts highlight the use of alternative methods, like the CUT system, to elucidate whether HPV-66 is underestimated due to the aforementioned difficulties in its detection or not, and also to corroborate its epidemiological classification and carcinogenicity.

The existence of intra-type variants with different oncogenic potential and precise patterns of geographic distribution has been documented for some HPV types, in particular for HPV-16 and -18 [Bernard et al., 2006]. However, the understanding of the mechanisms that give rise to new variants and types of HPV is still rudimentary. Since the identification of HPV-66, 10 variants have been described whose complete genomes are available at the GenBank. In addition, partial L1 gene sequences of about sixty putative HPV-66 variants have been identified in different geographic areas [Cerqueira et al., 2003; Prado et al., 2005; Castro et al., 2011; Wyant et al., 2011; Cento et al., 2012]. This study reports the first HPV-66 putative subtype and 7 novel putative variants (Fig. 3). However, the presence of additional variants in the 74 sequences with complete sequence identity to the known HPV-66 prototype and variants cannot be excluded. Indeed, the HPV-66 prototype (U31794.1) shows 100\% identity to HPV-66L1Qv25662 (EF177186.1) variant, and the HPV-66L1Qv35854 (EF177182.1) strain shares complete sequence identity to HPV-66L1Qv26488 (EF177184.1), HPV-66L1Qv24220 (EF177189.1), HPV-66L1Qv11088 (EF177190.1), and HPV-66L1Qv218 (EF177191.1) variants in the CUT amplicon. On the 
other hand, most of the putative HPV-66 variants previously described [Cerqueira et al., 2003; Prado et al., 2005; Castro et al., 2011; Wyant et al., 2011; Cento et al., 2012] are sequences obtained with the MY9/MY11 primers that do not overlap with the CUT amplicons. Further studies are required to assess the phylogenetic relationships of the novel HPV-66 strains reported here with respect to those variants/putative variants described previously. The characterization of the complete genomes of the novel putative subtype and of the variants found in this study will help to advance in the knowledge of the diversity and evolution of HPV-66. Moreover, the identification of differences and similarities between HPV-66 and other related HPVs might eventually shed light on what makes particular types trigger cancer, while others simply give rise to benign transformations. In conclusion, this study provides relevant and timely data on the pre-vaccine type-specific prevalence of HPV infection and associated-risk factors in sexually active women in Argentina which might be useful for the future assessment of the impact of massive vaccination. The high rates of HPV infection and the wide spectrum of types found in both groups of women support the need for the implementation of virological and molecular surveillance to monitor the epidemiological profile of HPV infection in the vaccine era. In this scenario, the ability demonstrated by the CUT primer system in detecting viruses from most species of the Alpha genus containing mucosal types suggests this testing strategy is a useful tool to explore the HPV types involved in cervix infections.

\section{Acknowledgments}

The authors thank Dr. Mercedes Leiva (Universidad Nacional de Rosario, Rosario, Argentina), and Dr. Debbie Indyk (Mount Sinai School of Medicine, New York, US) for helpful discussions.

This work was funded by the Agencia Nacional de Promoción Científica y Tecnológica (Grants PICT 05-25464 and PICT 2008-0421) and by a Fogarty International Center/NIH grant (Grant \# D43 TW 001037). The funders had no role in the study design, data collection and analysis, nor with the decision to publish or the preparation of the manuscript.

\section{References}

Abba MC, Gomez MA, Golijow CD. Human papillomavirus genotype distribution in cervical infections among woman in La Plata, Argentina. Rev Argent Microbiol. 2003; 35:74-79. [PubMed: 12920987]

Almonte M, Albero G, Molano M, Carcamo C, Garcia PJ, Perez G. Risk factors for human papillomavirus exposure and co-factors for cervical cancer in Latin America and the Caribbean. Vaccine. 2008; 26(Suppl 11):L16-36. [PubMed: 18945400]

Alliance for Cervical Cancer Prevention. Improving screening coverage rates of cervical cancer prevention programs: a focus on communities, Cervical Cancer Prevention Issues in Depth. Seattle: ACCP; 2004.

Arrossi S, Paolino M, Sankaranarayanan R. Challenges faced by cervical cancer prevention programs in developing countries: a situational analysis of program organization in Argentina. Rev Panam Salud Publica. 2010; 28:249-257. [PubMed: 21152712]

Bernard HU, Calleja-Macias IE, Dunn ST. Genome variation of human papillomavirus types: phylogenetic and medical implications. Int J Cancer. 2006; 118:1071-1076. [PubMed: 16331617]

Bosch FX, Burchell AN, Schiffman M, Giuliano AR, de Sanjose S, Bruni L, Tortolero-Luna G, Kjaer SK, Munoz N. Epidemiology and natural history of human papillomavirus infections and typespecific implications in cervical neoplasia. Vaccine. 2008; 26(Suppl 10):K1-16. [PubMed: 18847553]

Bruni L, Diaz M, Castellsague X, Ferrer E, Bosch FX, de Sanjose S. Cervical human papillomavirus prevalence in 5 continents: meta-analysis of 1 million women with normal cytological findings. $\mathrm{J}$ Infect Dis. 2010; 202:1789-1799. [PubMed: 21067372]

Castellsague X, Iftner T, Roura E, Vidart JA, Kjaer SK, Bosch FX, Munoz N, Palacios S, San Martin Rodriguez M, Serradell L, Torcel-Pagnon L, Cortes J. Prevalence and genotype distribution of 
human papillomavirus infection of the cervix in Spain: the CLEOPATRE study. J Med Virol. 2012; 84:947-956. [PubMed: 22499018]

Castro MM, Farias IP, Borborema-Santos CM, Correia G, Astolfi-Filho S. Prevalence of human papillomavirus (HPV) type 16 variants and rare HPV types in the central Amazon region. Genet Mol Res. 2011; 10:186-196. [PubMed: 21341210]

Cento V, Rahmatalla N, Ciccozzi M, Lo Presti A, Perno CF, Ciotti M. Human papillomaviruses 53 and 66: clinical aspects and genetic analysis. Virus Res. 2012; 163:212-222. [PubMed: 22001139]

Cerqueira DM, Camara GN, da Cruz MR, Silva EO, de Brigido MM, Carvalho LG, Martins CR. Variants of human papillomavirus types 53, 58 and 66 identified in Central Brazil. Virus Genes. 2003; 26:83-87. [PubMed: 12680696]

Chouhy D, Gil LB, Nocito AL, Wojdyla D, Ornella L, Cittadini J, Gardiol D, Giri AA. Development and evaluation of a colorimetric PCR system for the detection and typing of human papillomaviruses. Int J Mol Med. 2006; 18:995-1003. [PubMed: 17016633]

Chouhy D, Gorosito M, Sanchez A, Serra EC, Bergero A, Fernandez Bussy R, Giri AA. New generic primer system targeting mucosal/genital and cutaneous human papillomaviruses leads to the characterization of HPV 115, a novel Beta-papillomavirus species 3. Virology. 2010; 397:205216. [PubMed: 19948351]

da Silva MC, Martins HP, de Souza JL, Tognim MC, Svidzinski TI, Teixeira JJ, Consolaro ME. Prevalence of HPV infection and genotypes in women with normal cervical cytology in the state of Parana, Brazil. Arch Gynecol Obstet. 2012

de Sanjose S, Quint WG, Alemany L, Geraets DT, Klaustermeier JE, Lloveras B, Tous S, Felix A, Bravo LE, Shin HR, Vallejos CS, de Ruiz PA, Lima MA, Guimera N, Clavero O, Alejo M, Llombart-Bosch A, Cheng-Yang C, Tatti SA, Kasamatsu E, Iljazovic E, Odida M, Prado R, Seoud M, Grce M, Usubutun A, Jain A, Suarez GA, Lombardi LE, Banjo A, Menendez C, Domingo EJ, Velasco J, Nessa A, Chichareon SC, Qiao YL, Lerma E, Garland SM, Sasagawa T, Ferrera A, Hammouda D, Mariani L, Pelayo A, Steiner I, Oliva E, Meijer CJ, Al-Jassar WF, Cruz E, Wright TC, Puras A, Llave CL, Tzardi M, Agorastos T, Garcia-Barriola V, Clavel C, Ordi J, Andujar M, Castellsague X, Sanchez GI, Nowakowski AM, Bornstein J, Munoz N, Bosch FX. Human papillomavirus genotype attribution in invasive cervical cancer: a retrospective cross-sectional worldwide study. Lancet Oncol. 2010; 11:1048-1056. [PubMed: 20952254]

de Villiers EM, Fauquet C, Broker TR, Bernard HU, zur Hausen H. Classification of papillomaviruses. Virology. 2004; 324:17-27. [PubMed: 15183049]

Eklund C, Zhou T, Dillner J. Global proficiency study of human papillomavirus genotyping. J Clin Microbiol. 2010; 48:4147-4155. [PubMed: 20844222]

Ferlay J, Shin HR, Bray F, Forman D, Mathers C, Parkin DM. Estimates of worldwide burden of cancer in 2008: GLOBOCAN 2008. Int J Cancer. 2010; 127:2893-2917. [PubMed: 21351269]

Hosmer, D.; Lemeshow, S. Applied logistic regression. New York: Wiley; 2000.

Longworth MS, Laimins LA. Pathogenesis of human papillomaviruses in differentiating epithelia. Microbiol Mol Biol Rev. 2004; 68:362-372. [PubMed: 15187189]

Manos M, Ting Y, Wright D, Lewis A, Broker T, Wolinski S. Use of polymerase chain reaction amplification for the detection of genital human papillomaviruses. Cancer Cells. 1989; 7:209-214.

Matos E, Loria D, Amestoy GM, Herrera L, Prince MA, Moreno J, Krunfly C, van den Brule AJ, Meijer CJ, Munoz N, Herrero R. Prevalence of human papillomavirus infection among women in Concordia, Argentina: a population-based study. Sex Transm Dis. 2003; 30:593-599. [PubMed: 12897679]

Mazarico E, Gonzalez-Bosquet E. Prevalence of infection by different genotypes of human papillomavirus in women with cervical pathology. Gynecol Oncol. 2012; 125:181-185. [PubMed: 22226808]

Munoz N, Bosch FX, de Sanjose S, Herrero R, Castellsague X, Shah KV, Snijders PJ, Meijer CJ. Epidemiologic classification of human papillomavirus types associated with cervical cancer. $\mathrm{N}$ Engl J Med. 2003; 348:518-527. [PubMed: 12571259]

Prado JC, Calleja-Macias IE, Bernard HU, Kalantari M, Macay SA, Allan B, Williamson AL, Chung LP, Collins RJ, Zuna RE, Dunn ST, Ortiz-Lopez R, Barrera-Saldana HA, Cubie HA, Cuschieri K, von Knebel-Doeberitz M, Sanchez GI, Bosch FX, Villa LL. Worldwide genomic diversity of the 
human papillomaviruses-53, 56, and 66, a group of high-risk HPVs unrelated to HPV-16 and HPV-18. Virology. 2005; 340:95-104. [PubMed: 16039686]

Saiki RK, Bugawan TL, Horn GT, Mullis KB, Erlich HA. Analysis of enzymatically amplified betaglobin and HLA-DQ alpha DNA with allele-specific oligonucleotide probes. Nature. 1986; 324:163-166. [PubMed: 3785382]

Schiffman, M. Epidemiology of cervical human papillomavirus infections. In: zur Hausen, H., editor. Human pathogenic papillomaviruses Current topics in microbiology and immunology. Berlin: Springer Verlag; 1994. p. 55-81.

Schiffman M, Clifford G, Buonaguro FM. Classification of weakly carcinogenic human papillomavirus types: addressing the limits of epidemiology at the borderline. Infect Agent Cancer. 2009; 4:8. [PubMed: 19486508]

Solomon D, Davey D, Kurman R, Moriarty A, O’Connor D, Prey M, Raab S, Sherman M, Wilbur D, Wright T Jr, Young N. The 2001 Bethesda System: terminology for reporting results of cervical cytology. JAMA. 2002; 287:2114-2119. [PubMed: 11966386]

Tawheed AR, Beaudenon S, Favre M, Orth G. Characterization of human papillomavirus type 66 from an invasive carcinoma of the uterine cervix. J Clin Microbiol. 1991; 29:2656-2660. [PubMed: 1663515]

Villa LL. Overview of the clinical development and results of a quadrivalent HPV (types 6, 11, 16, 18) vaccine. Int J Infect Dis. 2007; 11(Suppl 2):S17-25. [PubMed: 18162241]

WHO/ICO. Information Centre on HPV and Cervical Cancer (HPV Information Centre). Human Papillomavirus and Related Cancers in Argentina. Summary Report 2010. 2010. www.who.int/ hpvcentre

Wyant PS, Cerqueira DM, Moraes DS, Leite JP, Martins CR, de Macedo Brigido M, Raiol T. Phylogeny and polymorphism in the long control region, E6, and L1 of human papillomavirus types 53, 56, and 66 in central Brazil. Int J Gynecol Cancer. 2011; 21:222-229. [PubMed: 21270605]

zur Hausen H. Papillomaviruses and cancer: from basic studies to clinical application. Nat Rev Cancer. 2002; 2:342-350. [PubMed: 12044010] 


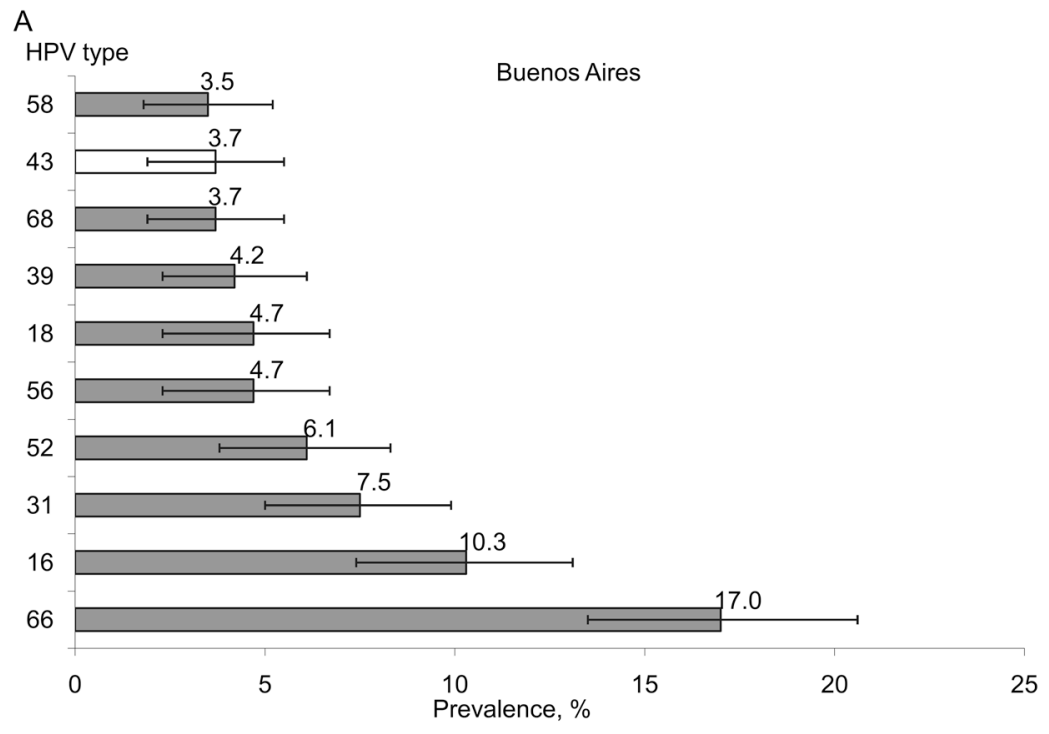

B

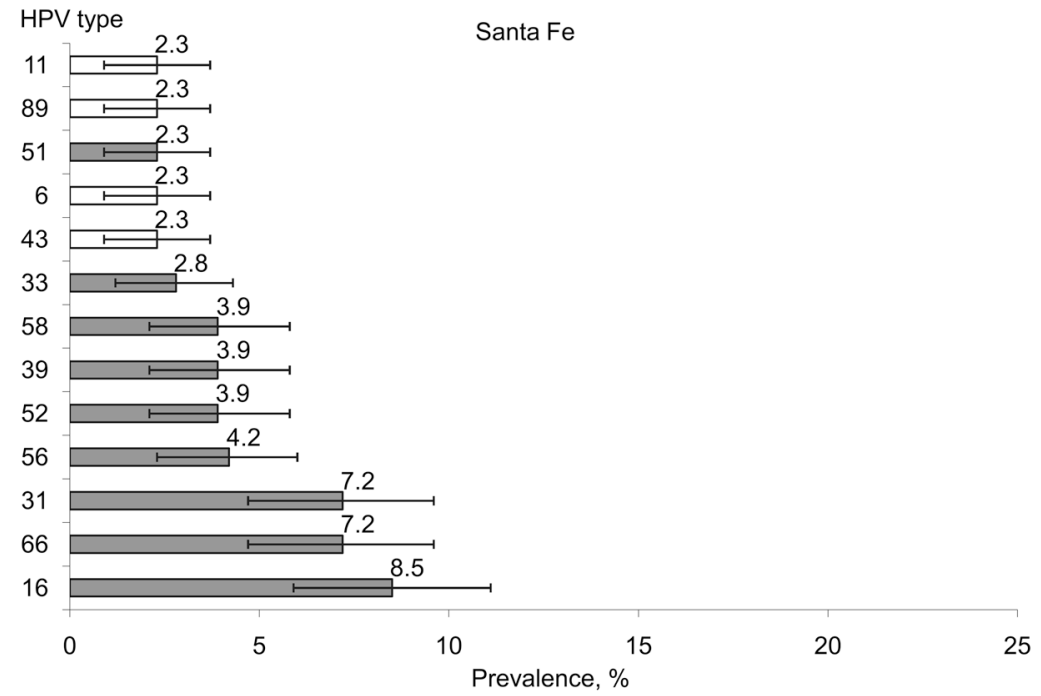

Fig. 1. Most frequent HPV types found in Buenos Aires (A) and Santa Fe (B) sites

Error bars indicate $95 \%$ confidence intervals. Grey bars and white bars indicate HR and LR HPV genotypes, respectively. 


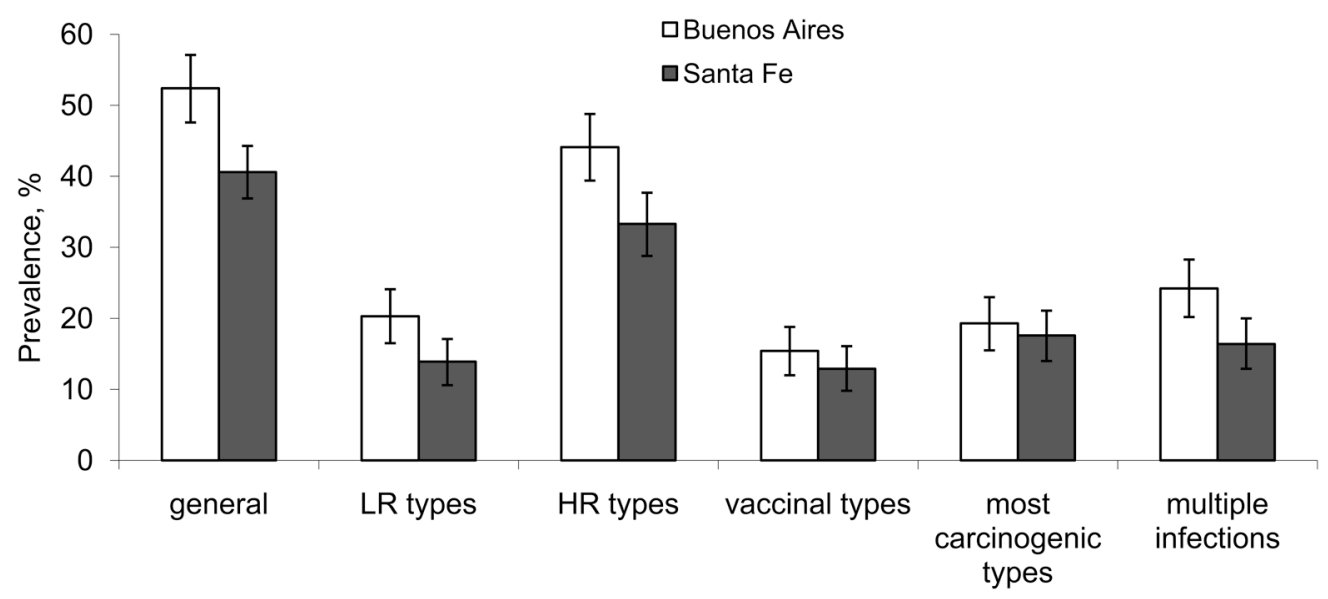

Fig. 2. Prevalence of HPV infection, specific types and multiple infections by site Vaccinal types refer to the presence of any of the types included in the quadrivalent vaccine against HPV (types $6,11,16,18$ ). Most carcinogenic types refer to the presence of at least one of the 5 viruses (HPV types 16, 18, 31, 33, 45) most frequently found in invasive cervical carcinomas [de Sanjose et al., 2010]. Error bars indicate 95\% confidence intervals. 
A

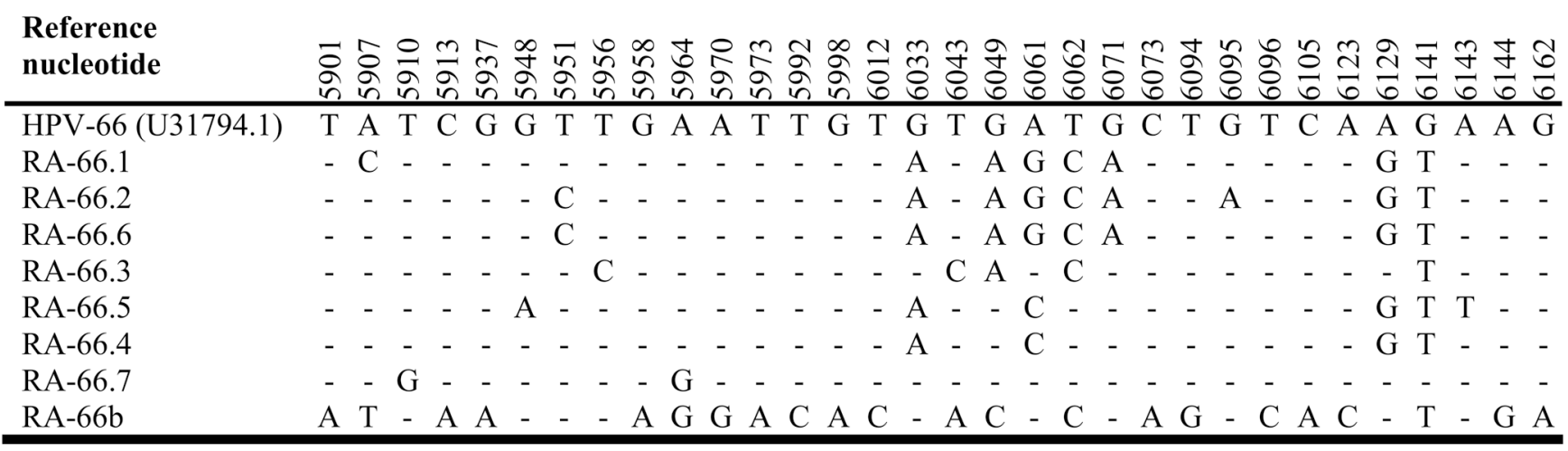

B

Reference HPV-66/variants

HPV-66 (U31794.1)

HPV-66L1Qv35854 (EF177182.1)

HPV-66L1Qv25111 (EF177188.1)

HPV-66L1Qv25260 (EF177187.1)

HPV-66L1Qv25696 (EF177185.1)

HPV-66L1Qv26740 (EF177183.1)

\section{Patient ID}

\section{Putative subtype/variants}

\begin{tabular}{cccccccc}
\hline RA-66b & RA-66.1 & RA-66.2 & RA-66.3 & RA-66.4 & RA-66.5 & RA-66.6 & RA-66.7 \\
\hline
\end{tabular}

$\begin{array}{llllllll}91.9 & 97.0 & 96.7 & 98.1 & 98.5 & 97.8 & 97.0 & 99.3\end{array}$

$\begin{array}{llllllll}91.1 & 99.6 & 99.3 & 97.8 & 98.5 & 97.8 & 99.6 & 96.7\end{array}$

$\begin{array}{llllllll}90.7 & 97.8 & 97.4 & 97.0 & 99.6 & 98.9 & 97.8 & 97.4\end{array}$

$\begin{array}{llllllll}90.7 & 99.3 & 98.9 & 97.4 & 98.1 & 97.4 & 99.3 & 96.3\end{array}$

$\begin{array}{llllllll}92.2 & 97.4 & 97.0 & 98.5 & 98.9 & 98.1 & 97.4 & 98.9\end{array}$

\begin{tabular}{llllllll}
90.7 & 98.1 & 97.8 & 97.0 & 99.3 & 98.5 & 98.1 & 97.4 \\
\hline
\end{tabular}

$\begin{array}{llllllll}\text { BA035 } & \text { SF311 } & \text { SF241 } & \text { SF067 } & \text { BA032 } & \text { BA032 } & \text { SF241 } & \text { BA101 } \\ & \text { SF352 } & \text { BA075 } & & \text { BA333 } & & \text { BA048 } & \text { BA292 } \\ & & & & & & \text { BA077 } & \end{array}$

BA080

BA137

Fig. 3. Genomic diversity of the novel HPV-66 putative subtype and variants

A. Nucleotide sequence variations among HPV-66 L1 sequences. L1 nucleotide positions where variations were observed are written across the top. Positions that do not vary from those of the HPV-66 prototype sequence are marked with dashes.

B. Pairwise nucleotide identities $(\%)$ of the novel putative subtype and variants with respect to reference HPV-66 strains. Only HPV-66 variants with complete genomes available at the GenBank database were considered as reference strains in the analysis. Patients from whom each novel putative subtype and variants were identified are indicated below. 


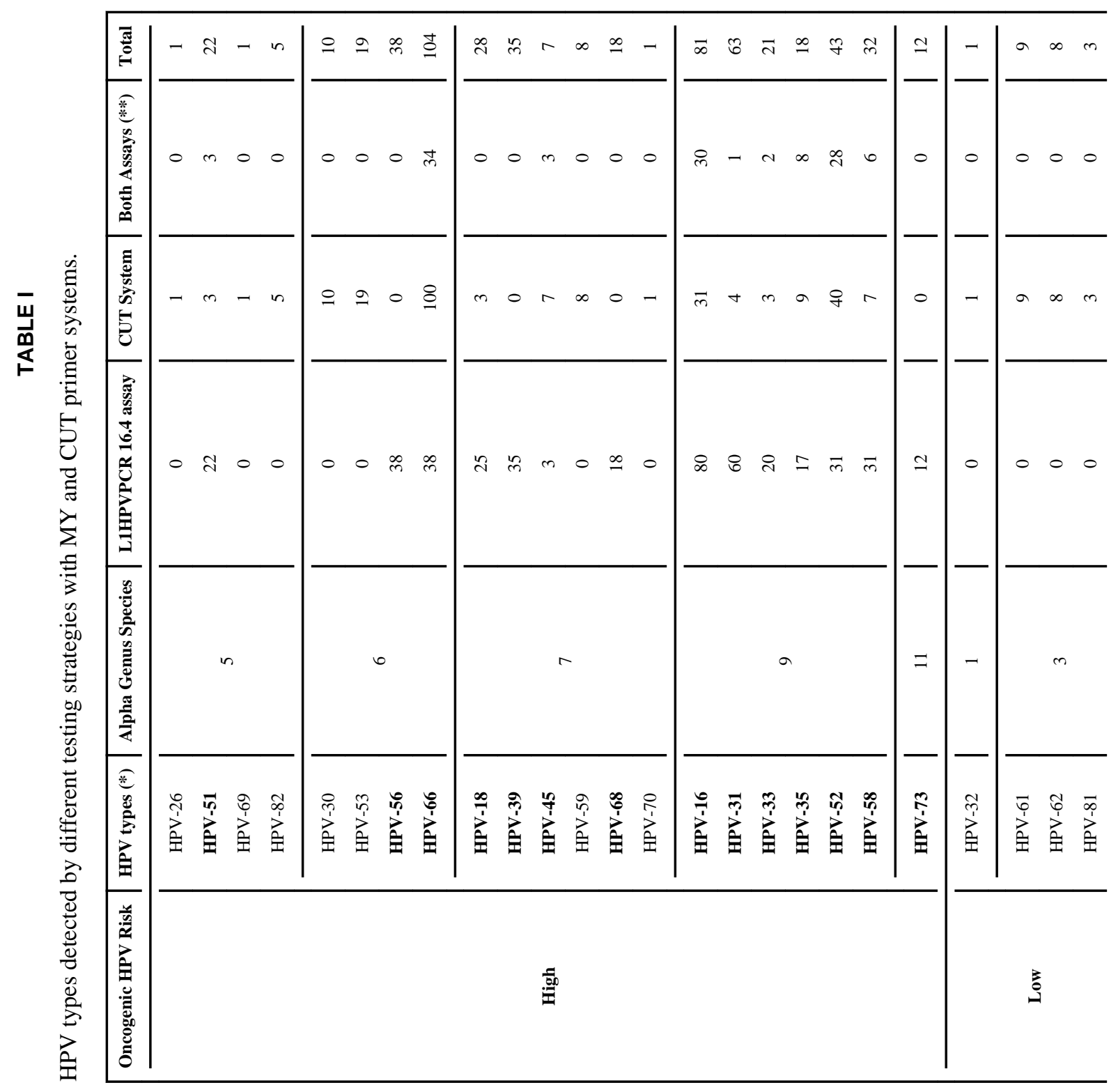

J Med Virol. Author manuscript; available in PMC 2014 April 01. 


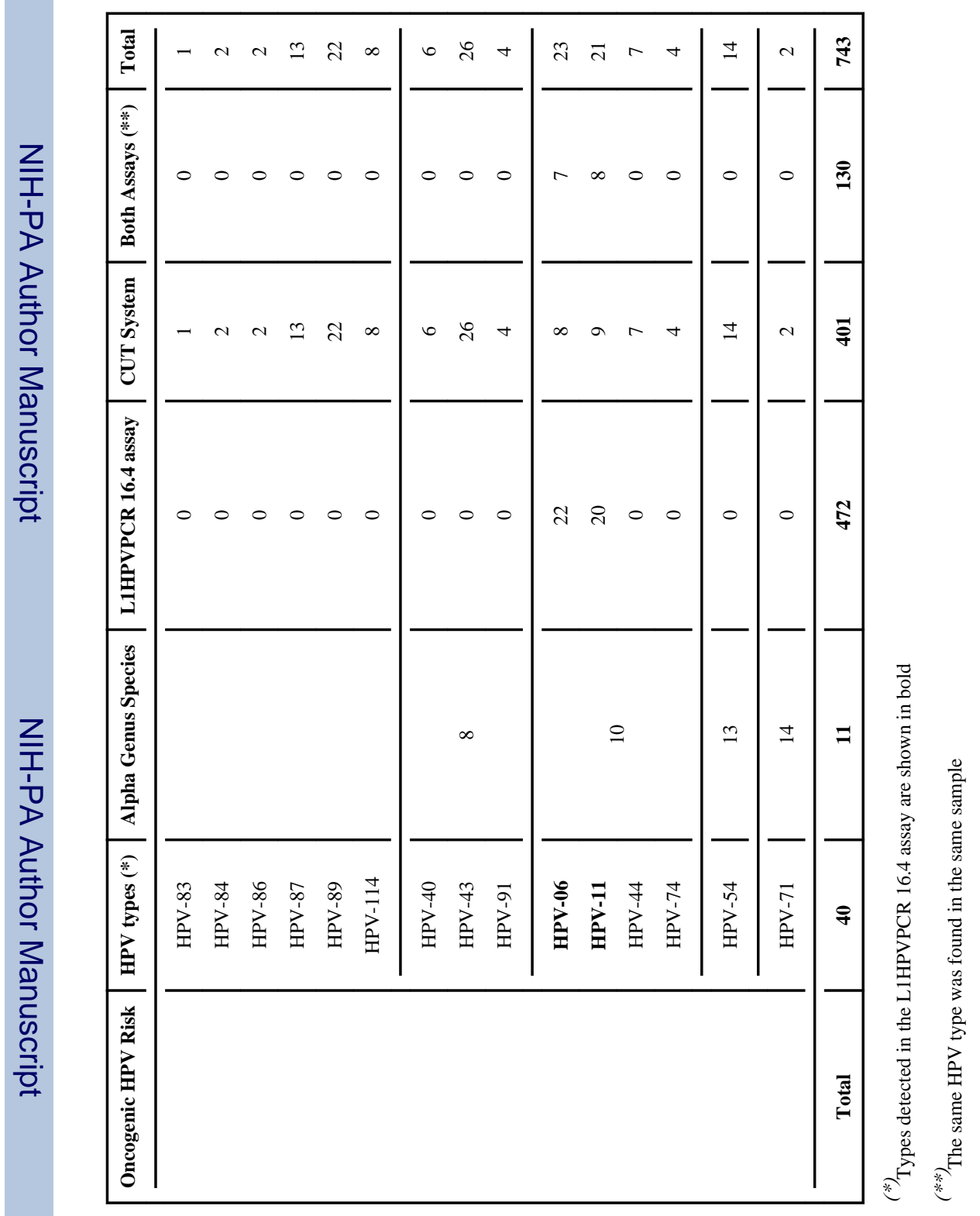




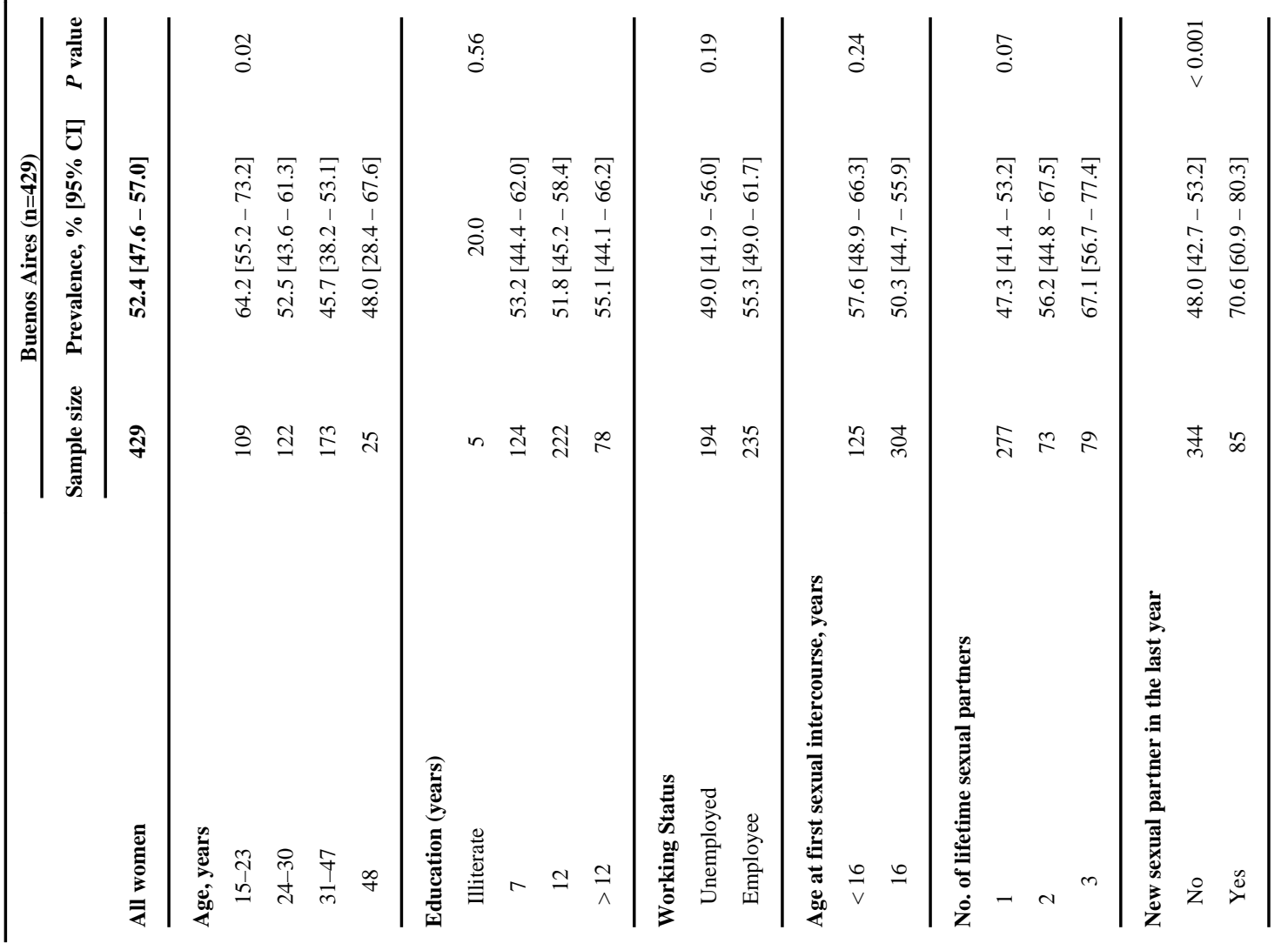




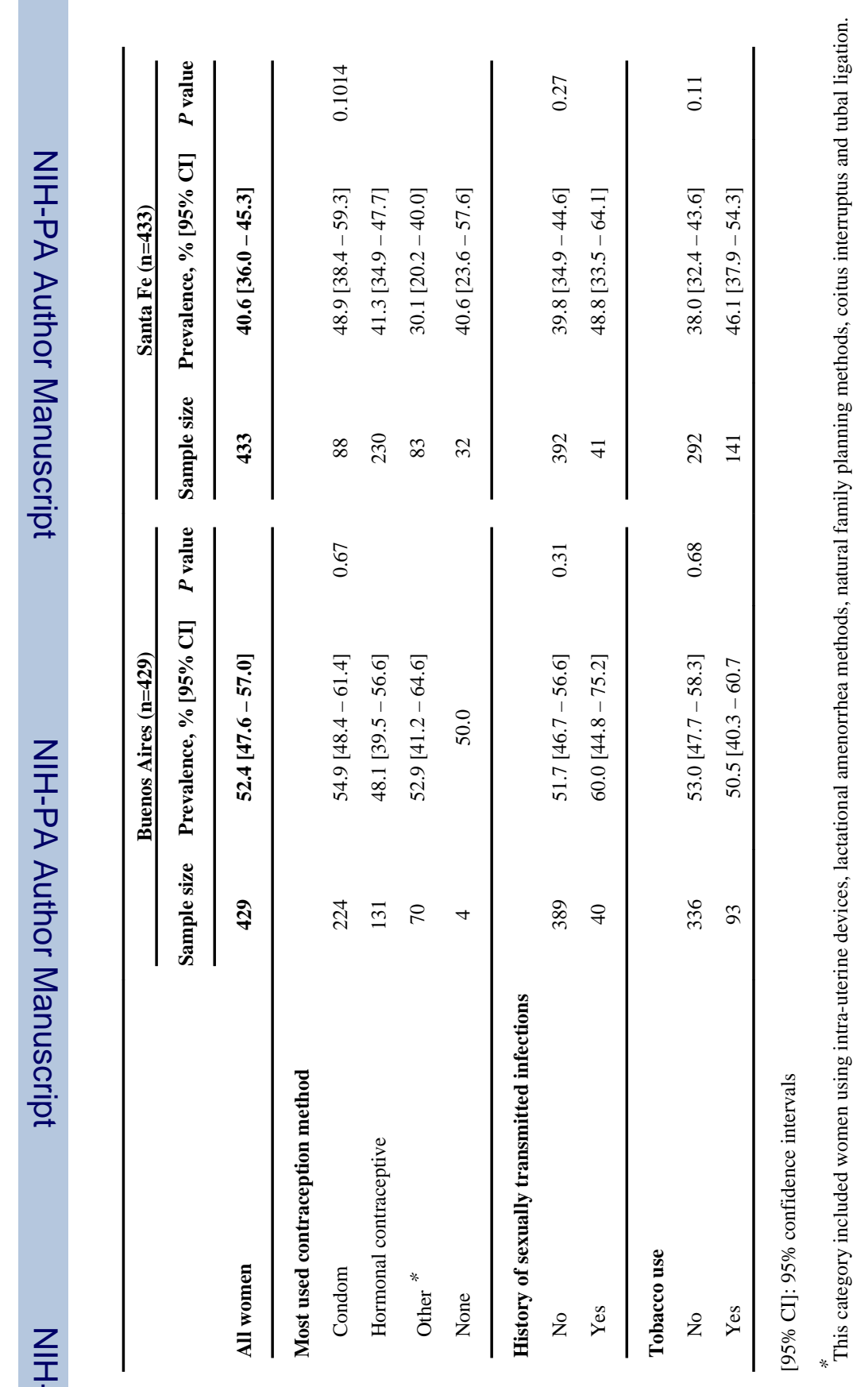




\section{TABLE III}

Multivariate analysis of variables associated with HPV infection.

\begin{tabular}{|c|c|c|}
\hline \multicolumn{3}{|c|}{ Buenos Aires $(n=429)$} \\
\hline & Odds ratio $[95 \% \mathrm{CI}]$ & $P$ value \\
\hline \multicolumn{3}{|c|}{ Age, years } \\
\hline $15-23$ & 1 (Referent) & \multirow{4}{*}{0.02} \\
\hline $24-30$ & $0.62[0.36-1.06]$ & \\
\hline $31-47$ & $0.48[0.29-0.79]$ & \\
\hline$\geq 48$ & $0.54[0.22-1.31]$ & \\
\hline \multicolumn{3}{|c|}{ New sexual partner in the last year } \\
\hline No & 1 (Referent) & \multirow{2}{*}{$<0.001$} \\
\hline Yes & $2.53[1.53-4.27]$ & \\
\hline \multicolumn{3}{|c|}{ Santa Fe $(n=433)$} \\
\hline & Odds ratio $[95 \% \mathrm{CI}]$ & $P$ value \\
\hline \multicolumn{3}{|c|}{ New sexual partner in the last year } \\
\hline No & 1 (Referent) & \multirow{2}{*}{0.04} \\
\hline Yes & $1.85[1.01-3.41]$ & \\
\hline
\end{tabular}

[95\% CI]: 95\% confidence intervals 


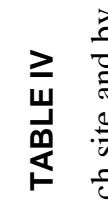

일

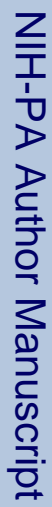

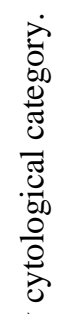

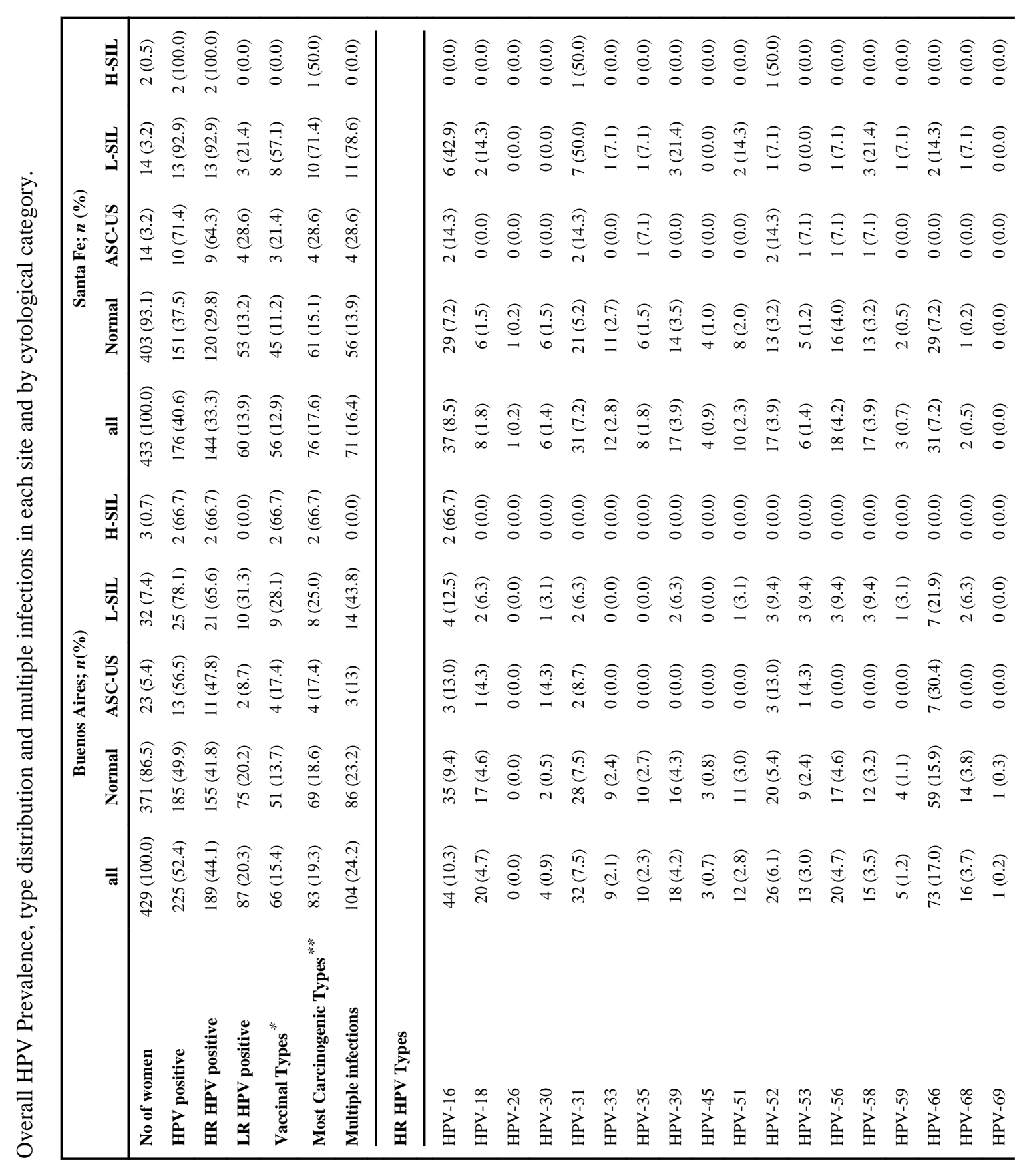

J Med Virol. Author manuscript; available in PMC 2014 April 01. 


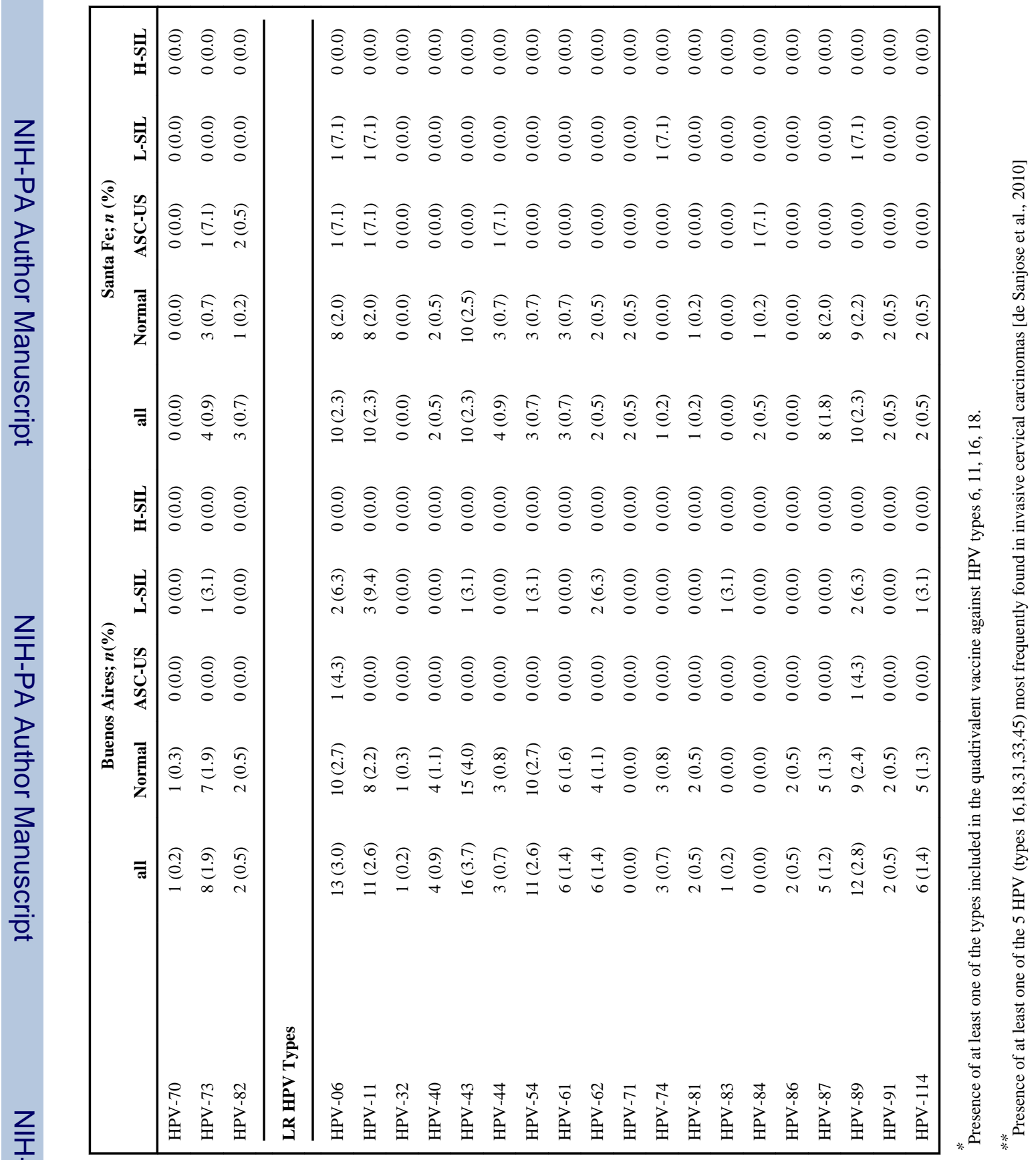

